\title{
Design and fabrication of an all-dielectric grating with top-hat high diffraction efficiency over a broad spectral range
}

\section{N. Lyndin}

\section{Flury}

\section{S. Tonchev}

R. Fechner

O. Parriaux

parriaux@univ-st-etienne.fr on leave from the Institute of General Physics Moscow, Russia

Laboratoire Hubert Curien UMR CNRS 5516 (formerly TSI), 18 rue B. Lauras, F-42000 Saint-Etienne

on leave from ISSP Sofia, Bulgaria

Institut für Oberflächenmodifizierung, IOM, Permoserstrasse 15, D-04318 Leipzig, Germany

Laboratoire Hubert Curien UMR CNRS 5516 (formerly TSI), 18 rue B. Lauras, F-42000 Saint-Etienne

Between $98 \%$ and $100 \%$ flat top efficiency $-1^{\text {st }}$ order TE diffraction over a $40 \mathrm{~nm}$ wavelength range centered at $800 \mathrm{~nm}$ can be obtained by an all-dielectric grating structure where the corrugation is etched in a high index layer unlike in the state of the art. $98 \%$ maximum efficiency and a wide-band top-hat diffraction efficiency spectrum are demonstrated experimentally. [D01: 10.2971/je0s.2007.07019]

Keywords: femtosecond laser, compression gratings, dielectric multilayer, leaky mode

\section{INTRODUCTION}

The last stage in a Chirped Pulse Amplification (CPA) scheme is recognized as the most critical and limiting one regarding flux resistance [1]. Standard femtosecond compression gratings are known to be limited in energy flux resistance [2]. They usually consist of a gold coating deposited onto a sinusoidal surface undulation replicated in some sort of resist [3]. High diffraction efficiency and fabricability requirements impose TM polarized incidence; this implies a large electric field at the metal surface and the risk of local plasmon excitation at surface imperfections. The resulting heat generation has to be dissipated essentially by the organic resist which leads to the degradation of the corrugation at less than $1 \mathrm{~J} / \mathrm{cm}^{2}$ fluence. This has motivated the search for an alternative as from the beginning of the nineties as first suggested by Sychugov et al. [4]. All-dielectric grating structures composed of a multilayer mirror and a corrugated superstructure have been developed in a number of laboratories [5, 6], and have been shown to withstand a fluence larger than $1 \mathrm{~J} / \mathrm{cm}^{2}$ at $1050 \mathrm{~nm}$ wavelength [7]. The possibility to achieve $100 \%$ diffraction efficiency was shown to be related with the excitation of a leaky mode of the mirror-based diffractive superstructure [8]. The polarization is preferably TE since the TM polarization exhibits the Brewster effect which limits the field accumulation in a leaky mode. Such gratings are today available commercially with impressive performances and size [9]. With the development of Ti:sapphire industrial lasers for machining applications in the $800 \mathrm{~nm}$ wavelength range [10], a new set of specifications has emerged with moderate bandwidth (about 30-40 $\mathrm{nm}$ ) and ultimate efficiency since the 4pass scheme currently used multiplies by about 4 the loss of a single diffraction event. Conventional all-dielectric gratings can reach $100 \%$ diffraction efficiency theoretically at a central wavelength where the leaky mode resonance is satisfied, but the efficiency slowly falls off at neighbouring spectral components. A bandwidth of typically $65 \mathrm{~nm}$ at $1064 \mathrm{~nm}$ wavelength at $95 \%$ diffraction efficiency is obtained in state of the art gratings where the corrugation is made in a low index silica top-layer [9]. A top-hat diffraction efficiency profile close to $100 \%$ over a restricted spectrum is needed for laser machining. We showed the way with an early sample exhibiting at some places $98 \%$ maximum diffraction efficiency, and an essentially top-hat spectrum of close to $20 \mathrm{~nm}$ width centered at $800 \mathrm{~nm}$ wavelength [11]. In the present paper the width of the flat top is stretched to $40 \mathrm{~nm}$ and the efficiency reaches between 96 and $98 \%$ efficiency over the whole grating area.

\section{GRATING DESIGN}

The basic grating structure is represented in Figure 1. It comprises a multilayer mirror on a substrate and a dielectric multilayer with a corrugation in the top high index layer at the air side unlike in the state of the art where it is etched in a low index silica layer [7]. The diffraction is produced by the sole$1^{\text {st }}$ order in a contradirectional scheme away from the Littrow condition. As shown in Ref. [12] the condition for high efficiency is given by the dispersion equation of a TE leaky mode excited by refraction of the incident wave in the corrugated layer.

Figure 1 also represents the rationale behind the high efficiency device. When the leaky mode resonance condition 


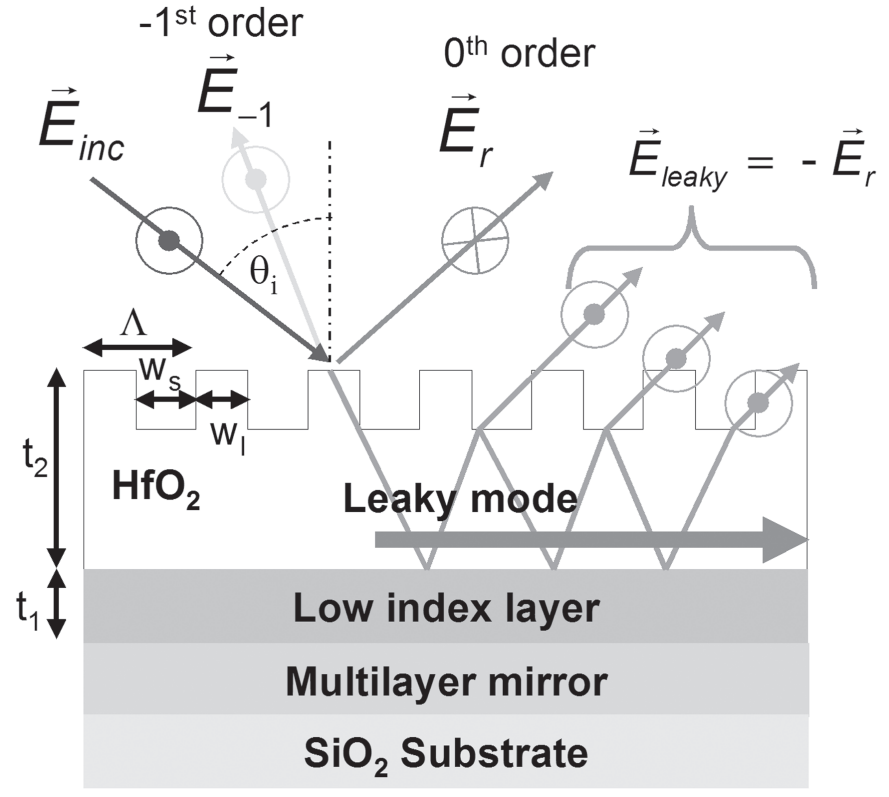

FIC. 1 Cross-sectional view of the dielectric mirror based leaky mode propagating structure with binary corrugation in the last high index layer. TE incidence is under angle $\theta_{i}$, diffraction along the sole $-1^{\text {st }}$ order. The Fresnel reflection is cancelled by balanced destructive interference between top reflected field and re-radiated leaky mode field. The circles with cross and dot represent the electric field with its orientation.

is fulfilled, the Fresnel reflection coefficient in a gratingless structure is real and the contribution to reflection of the air/layer interface and of the leakage of the field accumulating layer are of opposite sign. Degrading the quality factor of the field accumulating layer by means of $a-1^{\text {st }}$ order grating at the layer/air interface has two consequences: first, the Fresnel reflection can be cancelled out by destructive interference in the Fresnel reflection direction, secondly, the energy has nowhere else to propagate but to be diffracted along the $-1^{\text {st }}$ order. $100 \%$ diffraction efficiency is therefore ensured by properly adjusting the grating strength. It has long been believed that $100 \%$ diffraction efficiency in such non-Littrow configuration is a result of a condition of constructive interference in the incidence half-space between the wave directly diffracted by the grating and the wave diffracted down towards the mirror and reflected into the incidence half-space. The consequence of such assumption leads to a result which an exact electromagnetic modelling does not confirm [13]. If the leaky mode propagating structure on top of the mirror is composed of several layers, the same rationale applies. The leaky mode dispersion equation is more complex, but it is easy to derive as shown in Ref. [8]. In the present paper the leaky mode propagating structure is composed of a low index buffer and a high index top-layer into which the corrugation is etched. Exciting a leaky mode for enhancing the diffraction efficiency of a grating does not imply that the damage threshold of the grating is decreased. The electric field of a TE leaky mode is no larger than the electric field of the incident wave. Satisfying the leaky mode propagation condition only amounts to have a field maximum in the grating region and to enable a cancellation of the Fresnel reflection. Very different is the trapping of the field in a true guided mode where the field in the corrugated waveguide can be much larger than that of the inci- dent wave as in laser polarization control for instance [14] and leads to a significant decrease of the damage threshold [15].

Figure 2 shows the result of the modelling of the present structure.

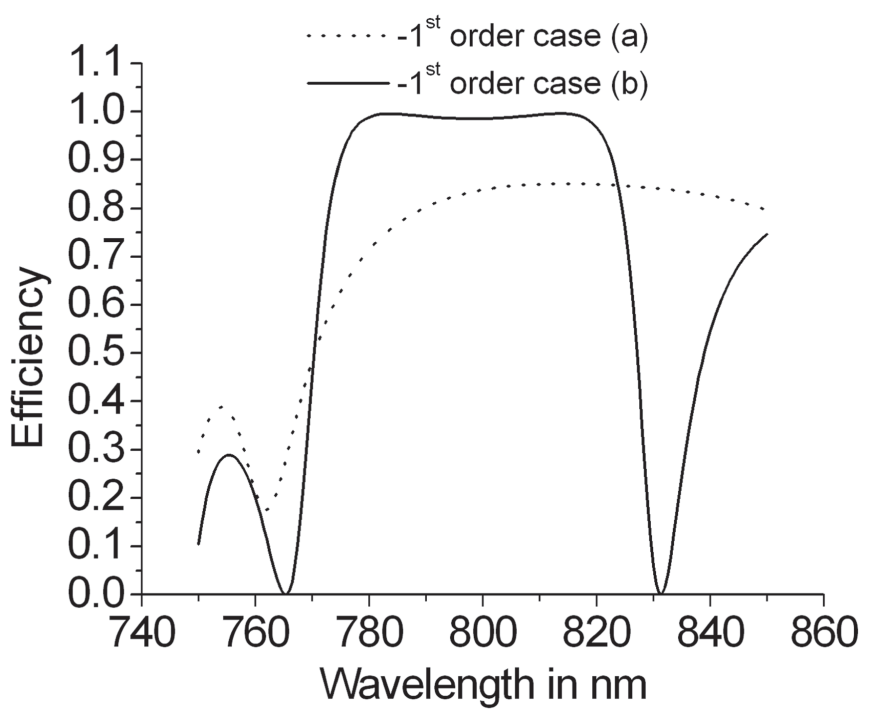

FIC. 2 - $^{\text {st }}$ order diffraction efficiency spectra: a) with corrugated two-layer structure of unity line/space ratio satisfying the leaky mode dispersion equation (modelling). The variables $t_{1}$ and $t_{2}$ obtained from equation (1) are respectively $30 \mathrm{~nm}$ and $110 \mathrm{~nm}$. The multilayer mirror contains 19 quarter-wave layers of $\mathrm{SiO}_{2}$ and $\mathrm{HfO}_{2}$. b) of the optimised broad band character.

Curve a) represents the $-1^{\text {st }}$ order diffraction efficiency spectrum of a typical all-dielectric structure comprising a quarterwave mirror of 19 layers, a low index buffer of thickness $t_{1}$ on top, and a high index corrugated top-layer of thickness $t_{2}$ at the air side. In the simple structure corresponding to curve a) the corrugation is etched all through the high index top-layer (the thickness of the non-etched part of the top-layer is zero) and the line width is equal to the groove width. The leaky mode propagating superstructure is consequently a two-layer system where the top-layer is corrugated and will be represented by an equivalent homogeneous layer in the leaky mode dispersion equation hereunder. The incidence angle in air is 57 degrees, the central wavelength is $800 \mathrm{~nm}$. The quarter-wave layers are made of silica $\left(n_{L}=1.48\right)$ and $\mathrm{HfO}_{2}\left(n_{H}=2.12\right)$ and are quarter-wave for the incident wave which is to be trapped in the leaky mode propagating superstructure, therefore $t_{L}=164 \mathrm{~nm}$, and $t_{H}=103 \mathrm{~nm}$. The mirror's last layer is of high index. The two-layer dispersion equation for the propagation of a TE leaky modes writes [8]

$$
\kappa_{2} \tan \left(\kappa_{2} t_{2}-\frac{\phi_{a}}{2}\right)+\kappa_{1} \tan \left(\kappa_{1} t_{1}-\frac{\phi_{m}}{2}\right)=0
$$

where $t_{1}$ is the thickness of the dielectric layer of index $n_{L}$ in direct contact with the multilayer mirror and $\kappa_{1}=$ $k_{0} \sqrt{n_{L}^{2}-n_{c}^{2} \sin ^{2} \theta_{i}}$ with $k_{0}=2 \pi / \lambda$ at vacuum wavelength $\lambda, \theta_{i}$ is the incidence angle in the incidence medium of index $n_{c}$, and $t_{2}$ is the thickness of the top-layer of high index $n_{H}$ where the corrugation will later be etched and $\kappa_{2}=$ $k_{0} \sqrt{n_{H}^{2}-n_{c}^{2} \sin ^{2} \theta_{i}}$. The phase terms $\phi_{m}$ and $\phi_{a}$ are the reflection phase shifts at the mirror boundary and at the air 
side with incidence from the leaky mode propagating layer side. $\phi_{a}$ is 0 since the transmission medium (air) has lower index, and $\phi_{m}$ is zero too since the last layer of the multilayer mirror is of high index. For the electric field to be strong in the high-index corrugation to ensure maximum strength with minimum depth, the dispersion Eq. (1) must be satisfied in a structure which is corrugated, therefore an equivalent index $n_{e q}$ must be used for the top layer instead of the high index $n_{H}$. In the case of the TE polarization the equivalent index is simply given by expressing the equality between the permittivity integrated over one grating period $\Lambda$ and the product of the layer thickness $t_{2}$ by the equivalent permittivity $\varepsilon_{e q}=n_{e q}^{2}$ :

$$
n_{e q}=\sqrt{\frac{w_{s}}{\Lambda}\left(\varepsilon_{c} \cdot \rho+\varepsilon_{H}\right)}
$$

and $\rho=w_{l} / w_{s}$ is the line/space ratio between the width $w_{l}$ of the grating line of permittivity $\varepsilon_{\mathcal{C}}=n_{c}^{2}$ and the groove width $w_{s}$ of permittivity $\varepsilon_{H}=n_{H}^{2}$.

The search for the conditions of maximum diffraction efficiency is first made by choosing two thicknesses $t_{1}$ and $t_{2}$ satisfying Eq. (1) approximately, then introducing the latter as the starting values into an optimization code. The diffraction efficiency profile is obtained by simply setting the thickness of the non-etched part of the top-layer to zero and the line/space ratio $\rho$ to 1 in a general diffraction modelling code based on the true-mode method [16]. Unlike the better known Rigorous Coupled Wave Analysis (or Fourier Modal Method) method [17], the true-mode method considers the grating corrugation as is, and develops the field on the basis of the true modes of the corrugation instead of developing the corrugation in a Fourier series. There is a plurality of combinations of $\rho, t_{1}, t_{2}$ and of the non-etched top-layer thickness satisfying Eq. (1) and achieving maximum diffraction efficiency; we chose for curve a) the simplest one ( $\rho=1$ and corrugation all through the top-layer) as a benchmark for further optimisation. The corresponding $-1^{\text {st }}$ order diffraction efficiency spectrum is shown as curve a) in Figure 2. It is not particularly wide band and does not always reach $100 \%$. It is not narrow band either since the maximum diffraction efficiency relies upon the excitation of a leaky mode which is a very broad resonance. A broad band top-hat character in the diffraction efficiency spectrum can be introduced by means of a specific tailoring of the multilayer dispersion. This will be reported separately. Submitting the full structure inclusive of the mirror to the optimisation code with the departure point of the structure corresponding to curve a) delivers the diffraction efficiency of curve b). The efficiency spectrum now exhibits a top-hat character with close to $100 \%$ over a broad band and quickly falls to zero at the band edges. Defining the corrugation in the last high index layer requires quite shallow grooves of hardly more than $100 \mathrm{~nm}$ depth whereas grooves defined in a silica layer must be between 400 and $600 \mathrm{~nm}$ deep to give rise to a sufficient grating strength [5]. Furthermore, a grating defined in a top low index layer can not provide such efficiency spectrum as given by curve b). The optimised structure giving rise to the diffraction efficiency spectrum of curve b) has a binary corrugation of $123 \mathrm{~nm}$ depth in the highindex hafnia layer of $253 \mathrm{~nm}$ thickness and the line/space ratio $\rho=220 / 350$ with a $570 \mathrm{~nm}$ period.

\section{EXPERIMENT}

The test structures were fabricated on thick fused quartz substrates of $25 \mathrm{~mm}$ diameter, $6 \mathrm{~mm}$ thickness by the deposition of alternating layers of silica and hafnia. The lithography of the $570 \mathrm{~nm}$ period grooves was made by exposing a $200 \mathrm{~nm}$ thick resist layer to the interferogram produced by a stabilized Mach-Zehnder scheme at the $442 \mathrm{~nm}$ wavelength of a HeliumCadmium laser. The problem of the reflection of the exposure beams by the multilayer is known to be a big problem, sometimes imposing a modification of the multilayer mirror [18]. The present structure is made to be little reflecting under specific exposure conditions which permits to skip any AntiReflection Coating (ARC) resist or absorptive layer, thus making the exposure on a high reflection mirror easy. The definition of narrow resist walls was made by a two-step exposure. Figure 3 illustrates the Atomic Force Microscope (AFM) scan of a resist grating exhibiting small line/space ratio $\rho$.

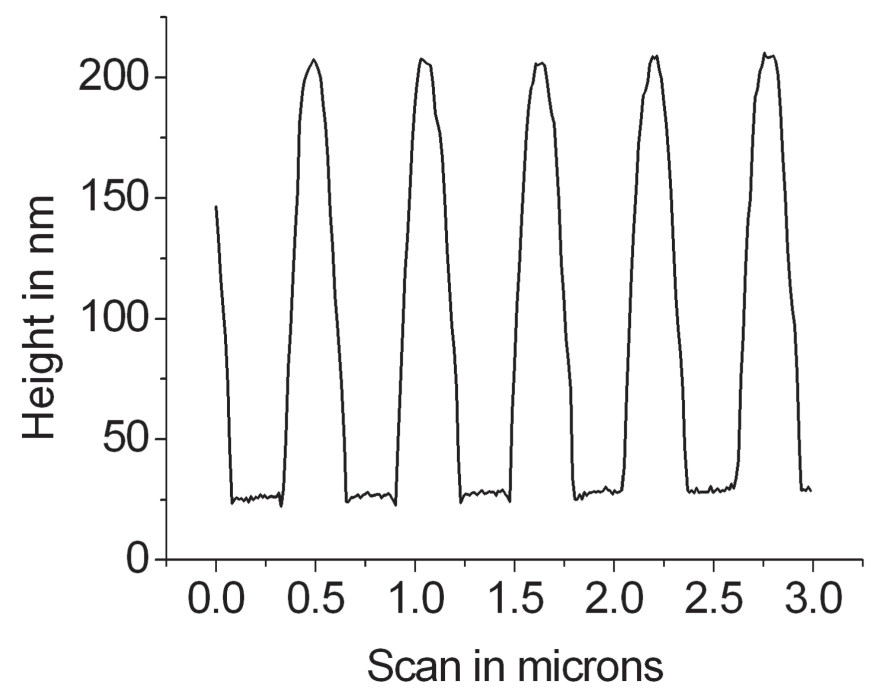

FIG. 3 AFM scan of a typical thin resist wall grating.

The physical transfer of the resist grating into the last hafnia layer was made by Reactive Ion Beam Etching (RIBE). Special care was taken not to broaden the corrugation walls. Figure 4 illustrates a typical corrugation obtained in hafnia which closely corresponds to the specifications given by the modelling with a groove depth of $123 \mathrm{~nm}$ and line/space ratio $\rho=0.62$.

The corrugated wafers were tested by means of a continuous wave tunable Ti:sapphire laser under an incidence angle of 57 degrees between 730 and $850 \mathrm{~nm}$ wavelength which was the available tuning range. As shown in Figure 5, the $-1^{\text {st }}$ order diffraction efficiency is remarkably high: $97 \%$ on the average and is remarkably flat between 777 and $815 \mathrm{~nm}$ wavelength. The $0^{\text {th }}$ order is $2 \%$ on the average and exhibits sharp peaks at the band edges. As compared with the theoretical modelling (doted curve of Figure 5) the experimental spectrum has a slight blue-shift of $3 \mathrm{~nm}$ which is attributed to an inaccurate layer index; for instance changing the index from 2.12 to 2.10 shifts the curve by $5 \mathrm{~nm}$ to shorter wavelengths. The experimental spectrum shows a slightly rounded left shoulder which is attributed to a small error on the line/space ratio 


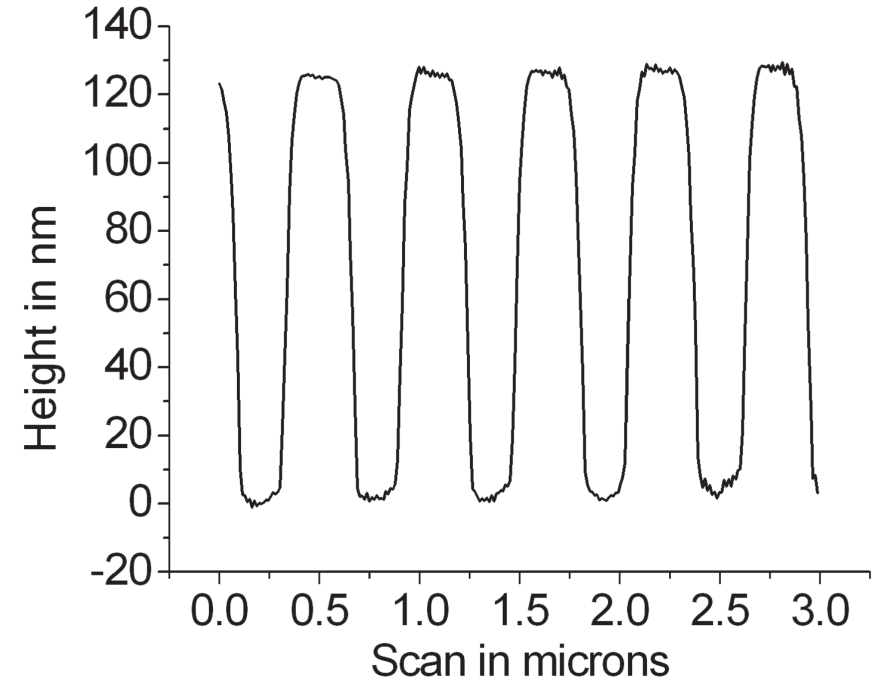

FIG. 4 AFM scan of the corresponding hafnia wall grating after RIBE.

$\rho$. A slightly larger line/space $\rho$ ratio will correct this effect. Making the measurement at different locations of the grating surface shows a few percent variation only which means that the technology can be scaled up to larger substrates. Although the diffraction efficiency is already quite high, these results do not represent a limit. There is room for improvement by a better control of the grating corrugation which appears to be the most sensitive element of the structure.

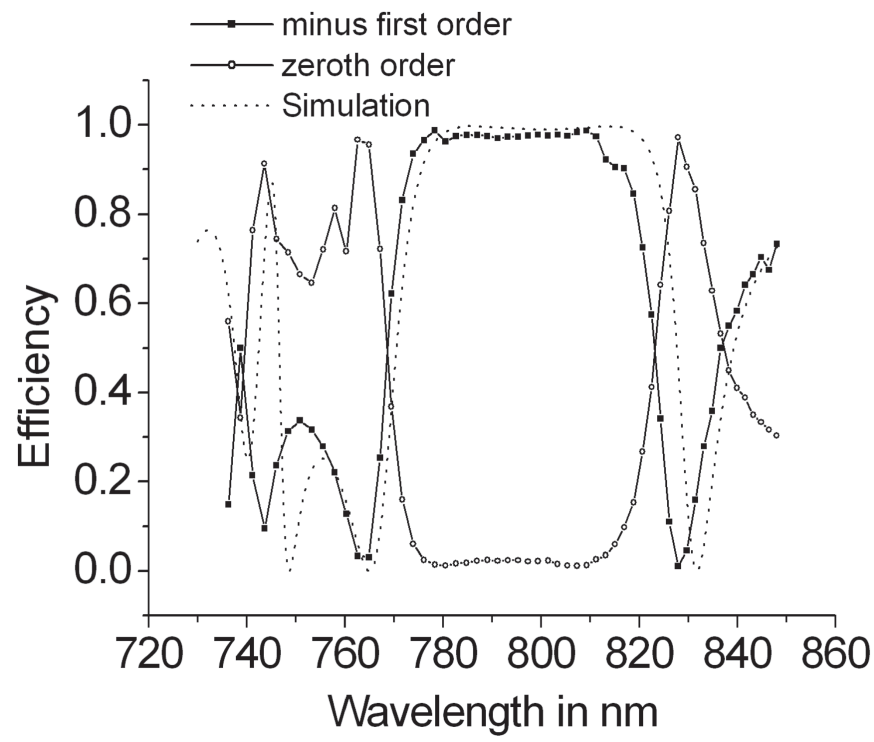

FIG. 5 Experimental diffraction efficiency spectrum under 57 degrees TE incidence. The dotted line corresponds to the modelled structure.

\section{CONCLUSION}

The evidence was shown that a compression grating of close to $100 \%$ efficiency over a $40 \mathrm{~nm}$ wide spectral width suitable for Ti:sapphire femtosecond laser machining of high average power can be fabricated with sufficient control. Unlike in the compression gratings of the state of the art, the corrugation is made in a layer of high refractive index which gives rise to high diffraction efficiency with a groove depth of hardly more than $100 \mathrm{~nm}$ and a groove duty cycle which does not differ much from 0.5 .

\section{ACKNOWLEDGMENTS}

The authors are grateful to the CNRS MRCT "FEMTO" for its support. A special thanks goes to Prof. J.P. Chambaret and Dr. F. Canova, ENSTA, for their constant encouragements and for preparing for the flux resistance tests.

They also want to thank Mrs S. Reynaud for her contribution in the AFM scans of the resist gratings, and Prof. A.V. Tishchenko for many enlightening discussions on the resonant mechanism governing the operation of such diffraction device.

\section{References}

[1] D. Strickland and G. Mourou, "Compression of amplified chirped optical pulses" Opt. Commun. 56, 219-221 (1985).

[2] A. Reichart, N. Blanchot, P. Y. Baures, H. Bercegol, B. Wattelier, J. P. Zou, C. Sauteret and J. Dijon, "CPA compression gratings with improved damage performance" Proc. SPIE 4347, 521-527 (2001).

[3] R. D. Boyd, J. A. Britten, D. E. Decker, B. W. Shore, B. C. Stuart, M. D. Perry and Lifeng Li, "High-efficiency metallic diffraction gratings for laser applications" Appl. Optics 34, 1697-1706 (1995).

[4] A. S. Svakhin, V. A. Sychugov and A. E. Tikhomirov, "Efficient diffraction elements for TE-polarized waves" Sov. Phys. Tech. Phys. 36, 1038-1040 (1991).

[5] J. A. Britten, M. D. Perry, B. W. Shore, R. D. Boyd, G. E. Loomis and R. Chow, "High efficiency dielectric multilayer gratings optimized for manufacturability and laser damage threshold" Proc. SPIE 2714, 511-520 (1996).

[6] K. Hehl, J. Bischoff, U. Mohaupt, M. Palme, B. Schnabel, L. Wenke, R. Bödefeld, W. Theobald, E. Welsch, R. Sauerbrey and H. Heyer, “High-Efficiency Dielectric Reflection Gratings: Design, Fabrication, and Analysis" Appl. Optics 38, 6257-6271 (1999).

[7] J. Néauport and N. Bonod, “Design, optimization and development of pulse compression gratings for the MPW-HE LIL" J. Phys. IV France 133, 669-672 (2006).

[8] M. Flury, A. V. Tishchenko and 0. Parriaux, "The leaky mode resonance condition ensures $100 \%$ diffraction efficiency of mirror based resonant gratings" IEEE J. Lightwave Technol. to be published, (July 2007).

[9] B. Touzet and J. R. Gilchrist, "Multilayer dielectric gratings enable more powerful high energy lasers" Photon. spectra 68-75 (2005).

[10] A. Ostendorf, T. Bauer, F. Korte, J. R. Howorth, C. Momma, N. H. Rizvi, F. Saviot, and F. Salin, "Development of an industrial femtosecond laser micromachining system" Proc. SPIE 4633, 128-135 (2002).

[11] F. Canova, J. P. Chambaret, 0. Uteza, P. Delaporte, M. Tondusson, E. Freysz, O. Parriaux, M. Flury, S. Tonchev and N. Lyndin, " $>97 \%$ top-hat efficiency, $>4 \mathrm{~J} / \mathrm{cm}^{2}$ damage threshold compression gratings" Proc. ICUIL (Sept. 2006).

[12] A. V. Tishchenko and V. A. Sychugov, "High grating efficiency by energy accumulation in a leaky mode" Opt. Quant. Electron. 32, 1027-1031 (2000). 
[13] A. S. Svakhin, V. A. Sychugov, A. E. Tikhomirov, "Diffraction gratings with high optical strength for laser resonators" Quantum Electron. 24, 233-235 (1994).

[14] M. Abdou Ahmed, F. Pigeon, A. V. Tishchenko, 0. Parriaux, Y. Ouerdane, S. Reynaud, J. C. Pommier and J. Fuchs, "Polarizing grating coupler for high Q laser cavities" IEEE J. Quantum. Elect. 39, 614619 (2003).

[15] J. F. Bisson, O. Parriaux, N. Destouches, A. V. Tishchenko and K. Ueda, " $99 \%$ diffraction efficiency leaky mode sustained resonant gratings and susceptibility to laser damage" Lasers and ElectroOptics, CLEO/Pacific Rim 2005, Tokyo, 435-436 (2005).
[16] M. Foresti, L. Menez and A. V. Tishchenko, "Modal method in deep metal-dielectric gratings: the decisive role of hidden modes", J. 0pt. Soc. Am. A 23, 2501-2509 (2006).

[17] P. Lalanne and G. M. Morris, "Highly improved convergence of the coupled-wave method for TM polarization" J. Opt. Soc. Am. A 13, 779-784 (1996).

[18] B. W. Shore, M. D. Perry, J. A. Britten, R. D. Boyd, M. D. Feit, H. T. Nguyen, R. Chow, G. E. Loomis and L. Li, "Design of highefficiency dielectric reflection gratings" J. Opt. Soc. Am. A 14, 12241237 (1997). 